\title{
Modelling and experimental characterisation of self-regulating resistive heating elements for disposable medical diagnostics devices
}

\author{
T. Pardy ${ }^{1}$, T. Rang ${ }^{1} \&$ I. Tulp ${ }^{2}$ \\ ${ }^{1}$ Thomas Johann Seebeck Department of Electronics, \\ Tallinn University of Technology, Estonia \\ ${ }^{2}$ SelfD Technologie GmbH, Germany
}

\begin{abstract}
Resistive heating elements based on positive temperature coefficient (PTC) ceramics have the inherent advantage of self-regulating their temperature output via a rapid increase in resistance with increasing temperatures, and therefore require no additional control circuitry. This is particularly advantageous for medical devices, especially disposable molecular diagnostics, where minimal space requirements and power dissipation are crucial design factors, as well as low cost per device. However, rapid prototyping and design validation in silico through finite element analysis are hindered by the scarcity of material data on commercially available heating elements. The simplest characterization of the function of these heating elements is through the temperature coefficient of resistance, which is easily measured yet still provides an acceptable approximation. We propose a finite element model that relies only on the temperature coefficient to simulate the function of PTC heating elements. Model validation was done using a commercially available PTC heating element, the temperature coefficient of which was measured and inputted into the model to simulate a PMMA test structure designed to house the heater and accept Lab-ona-Chip devices with typical dimensions ( $25 \mathrm{~mm} \times 75 \mathrm{~mm})$.

Keywords: positive temperature coefficient, lab-on-a-chip, resistive heating, microfluidics, computer aided design, finite element modelling.
\end{abstract}




\section{Introduction}

In molecular diagnostics, temperature control is required for a wide variety of assays, particularly immunoassays and nucleic acid amplification assays. These assays require complex liquid handling, usually done via microfluidics. For a portable, disposable device, it is necessary to have a heating solution with low power dissipation, minimal space requirements and low cost per device. These criteria are easily satisfied by electrical heaters, however, including control circuitry increases development costs and complexity, while having a negative effect on the robustness of the device. For a point-of-care test intended for use at home, minimal complexity is essential to rule out possible errors. To avoid the need for control circuitry, two options are available: one is that of PTC (positive temperature coefficient) ceramic pellets, the other is using PTC resistance wires. However, the topic of integrated, portable (preferably disposable) electrical microheaters for microfluidics has not been researched extensively according to the available literature.

The group of Wyzkiewicz et al. [1] proposed a self-regulating heating solution for microfluidics that utilises PTC resistance wires. One of their demonstration setups used printed circuit boards (PCB) as substrate, consisting of interdigitated tracks of carbon-copper-silver, which could be a reasonably cheap microheating solution for disposable molecular diagnostics. The demonstrated setup had an output temperature range of $30-70^{\circ} \mathrm{C}$, suitable for a wide variety of assays. However, looking at microheating solutions from an industrial perspective, developing a custom-tailored heating solution may be less cost-efficient than choosing a commercially available one and adapting the device geometry and parameters accordingly. In any case, several prototyping stages and device optimization are necessary, and the higher the number of steps, the more costly development is. To reduce these costs, in silico prototype validation may be employed, using finite element models to simulate and predict device behaviour for a particular design. This approach, coupled with computer aided design may significantly save on time and human labour during development.

The goal of the work described in this paper was to propose an option for the finite element modelling of microheating in disposable molecular diagnostics devices using self-regulating heaters. The proposed model was validated via a commercially available PTC heating element in an experimental setup imitating a microfluidics-based molecular diagnostics device. The finite element model relied on properties easily measured without extensive knowledge about the materials and internal structure of the heating element that would require disassembly.

\section{Theoretical background}

\subsection{Self-regulating heating elements}

In the self-regulating heating elements discussed in this paper, the positive temperature coefficient of resistance (PTCR) effect is used for the precise regulation of heat output. This effect means a rapid increase in resistance with 
temperature, which essentially regulates the current passing through the heating element. Although most of the manufacturers of currently commercially available heaters do not disclose material data, the most typically used material is doped (3or 5 -valent dopants) polycrystalline barium titanate $\left(\mathrm{BaTiO}_{3}\right)$ ceramic, mentioned in detail by Shioi et al. [2].

The onset of the PTCR effect is at the Curie temperature $\left(T_{c}\right)$ of the material, the transition temperature from ferroelectric to paraelectric phase. This temperature can be adjusted by selecting the proper dopant, such as strontium or lead as detailed by Hybrechts et al. [3], in turn determining the onset of the PTCR effect, and thus, the set point (or temperature range) of the heating element.

In order to characterise PTC heaters and the PTCR effect in practice, only the temperature coefficient of resistance $\alpha[1 / \mathrm{K}]$ and the initial resistance $R_{0}[\Omega]$ are needed, but the latter is measured during recording the data points necessary for calculating the temperature coefficient, where $\alpha$ may be perceived as the slope of the linear curve with which the resistance of the PTC material is approximated.

\subsection{Finite element models}

\subsubsection{Simplified model}

The basis for modelling resistive heating is provided by Joule's first law, which states that electric currents passing through a conductor generate heat, which is proportional to the resistance of the conductor $R[\Omega]$ and the current $I[A]$ passing through it:

$$
Q \propto I^{2} \cdot R
$$

$Q[W]$ is the energy (heat) released, and if direct current is applied, can be expressed via the formula of power: $P=V \cdot I=I^{2} \cdot R$. This alone is enough to build a simplified model of electrical heating, if the assumption is made that the whole heating element uniformly releases the same amount of heat over its whole volume. This is valid if the size of the heater is small enough, and the heating element itself is made for a uniform power distribution. Additionally, the assumption is made that liquid in the microchannel is either slow enough to be considered stationary, or is kept stationary for the duration of heating. Therefore the model consists of two equations: heat generation is expressed by the formula of power $\left(P=I^{2} R\right)$, and then heat is propagated through the geometry of the model by the heat transfer equation, eqn. (4). Space-dependence in the model is defined by a Cartesian coordinate system with 3 axes $x[m], y[m], z[m]$, whereas time-dependence is described by time $(t)$ in seconds. Scaling is permitted. Heat generation is expressed via the aforementioned formula of power, which is interpreted for the whole volume of the heater (von Meier [4]):

$$
Q=\frac{P_{t o t}}{V}
$$

where $P_{t o t}[W]$ is the total power of the heater, $\mathrm{V}\left[\mathrm{m}^{3}\right]$ the volume of the heating element. In case the temperature dependence of resistance is taken into account, 
the well-known formula for calculating temperature dependent resistance (or resistivity) defines resistance changes (Rood [5]):

$$
R(T)=R_{0} \cdot\left[1+\alpha \cdot\left(T-T_{0}\right)\right]
$$

where $R_{0}[\Omega]$ is the resistance at ambient (reference) temperature $T_{0}[K] . T[K]$ is the current temperature of the heating element, calculated from the heat transfer equation. $\alpha\left[\frac{1}{K}\right]$ is the temperature coefficient of resistance. The heat transfer equation is used in the following form (Kandlikar [6]):

$$
\rho C_{p} \frac{\partial T}{\partial t}-\nabla \cdot(-\mathrm{k} \nabla \mathrm{T})=\mathrm{Q}
$$

where $\rho\left[\mathrm{kg} / \mathrm{m}^{3}\right]$ is the fluid density, $C_{p}[J / \mathrm{kg} \mathrm{K}]$ the specific heat capacity at constant pressure, $T[K]$ the absolute temperature in the system, $k[W /(m K)]$ the thermal conductivity, and Q $\left[W / \mathrm{m}^{3}\right]$ is the heat source (sink), which in this case is calculated as the power of the heating element as a whole. Materials of the simulated geometry are simulated through the aforementioned quantities $\left(\rho, C_{p}, k\right)$, solved for each spatial point in the geometry, whereas the heating element is characterized by its temperature coefficient of resistance $(\alpha)$.

\subsubsection{Extended model}

In case the space-dependence of heat generation cannot be neglected, either due to the comparable size of components, or due to the complex structure of the heating element itself, the model introduced earlier must be extended. First, Joule heating (introduced in eqn. (1)) must be rewritten in a differential form for the volume of the heater (von Meier [4]):

$$
\frac{d P}{d V}=J \cdot E=J \cdot \frac{J}{\sigma}=J^{2} \cdot \rho
$$

where $P[W]$ is once more the power, $\mathrm{V}\left[\mathrm{m}^{3}\right]$ the volume of the element, $\mathrm{J}\left[\mathrm{A} / \mathrm{m}^{2}\right]$ the current density, $\mathrm{E}[\mathrm{V} / \mathrm{m}]$ the electric field. $\sigma[\mathrm{S} / \mathrm{m}]$ is the conductivity, $\rho[\Omega \cdot m]$ is the resistivity of the material of the heater. Heat propagation relies on the same heat transfer equation (eqn. (4)), but heat generation is expressed as follows (Griffiths [7]):

$$
\begin{gathered}
\nabla J=Q_{j}, \\
J=\left(\sigma+\varepsilon_{0} \varepsilon_{r} \frac{\partial}{\partial t}\right) E+J_{e}, \\
E=-\nabla V .
\end{gathered}
$$

where $\mathrm{J}\left[A / \mathrm{m}^{2}\right]$ is the current density, $Q_{j}\left[A / \mathrm{m}^{3}\right]$ are current sources (sinks), E the electric field, $J_{e}\left[A / \mathrm{m}^{2}\right]$ an external current density (if there is one), $V[V]$ the potential drop in every direction, $\sigma[\mathrm{S} / \mathrm{m}]$ the conductivity, $\varepsilon_{0}$ the relative permittivity of vacuum, $\varepsilon_{r}$ the relative permittivity of the material at the point of 
the geometry for which the equations are solved. Materials in the simulated geometry are therefore characterized electrically through their conductivity and relative permittivity. This set of equations relies on the continuity equation, or charge conservation principle:

$$
\nabla J=-\frac{\partial p}{\partial t}
$$

The continuity equation states that charge density at a point only changes if current flows into or out of the point. Due to the fact, that heating elements with a positive temperature coefficient of resistance have a non-linear resistivity with respect to temperature, the resistivity must be calculated during simulation at each time instant from eqn. (3), substituting resistivity for resistance (conductivity: $\sigma=$ $1 / \rho)$. Resistivity at ambient temperature $\left(\rho_{0}[\Omega \cdot m]\right)$ is possible to calculate from the resistance at ambient temperature, extracting surface area and thickness of the heating element from the model geometry.

\section{Experimental setup for model validation}

For the experimental validation, a commercially available self-regulating heating element was used. The DBK HP04-1/04-24 heater plate consists of a PTC ceramic heating element, and an aluminium profile with a flat surface on one side. The operating range of this heater was $1-30 \mathrm{~V}_{\mathrm{DC}}$, and its highest measured temperature output was $65.1^{\circ} \mathrm{C}$.

The experimental setup consisted of a PMMA (polymethyl methacrylate) test structure designed to house the heating element and the temperature probe adapter, a DC multimeter (Agilent 34410A) and a DC power supply (Agilent E3631A). The temperature probe adapter was designed to imitate the typical size regime of microfluidic chips $(25 \mathrm{~mm} \times 75 \mathrm{~mm}$ ), and contained a $2 \mathrm{~W}$ thermistor with $10 \mathrm{k} \Omega$ initial resistance. The adapter was inserted into the slot in the PMMA frame, positioned as close to the flat face of the aluminium profile of the heating element as possible. The assembled test structure is shown in Figure 1(A).


Figure 1: Test structure (A) and model geometry (B). 


\section{Results and discussion}

\subsection{Electrical characterization of heating element}

Measurements were conducted using the previously described experimental setup, recording the current flowing through the heating element, and calculating resistance using Ohm's law. The temperature coefficient of resistance calculated with eqn. (3) was $0.23 \pm 0.09[1 / \mathrm{K}]$.

The resistance of the heating element at ambient temperature (considered $20^{\circ} \mathrm{C}$ in this case) was measured and calculated to be $10 \Omega( \pm 0.4 \Omega)$.

\subsection{Evaluation of the simplified model}

Most isothermal nucleic acid amplification methods require heating for an extended period of time with adequate temperature stability, typically at target temperatures less than $100^{\circ} \mathrm{C}$. For the following evaluation, to prevent accidental heat deflection of the plastic test structure, the maximum temperature was limited at $70^{\circ} \mathrm{C}$. Room temperature was defined as the range of $19-22^{\circ} \mathrm{C}$, corresponding to the average ambient temperature range in the room where measurements were performed.

The simplified model was solved using the Heat Transfer Module of COMSOL $^{\circledR}$ Multiphysics Version 4.4 for the model geometry shown in Figure 1(B). Materials were either used from the built-in libraries of the software package, or extracted from corresponding literature, such as the work of Shioi et al. [2]. To make a comparison, the model was solved for the same input voltages as were given for the experimental setup, ranging from $5 \mathrm{~V}-25 \mathrm{~V}$.

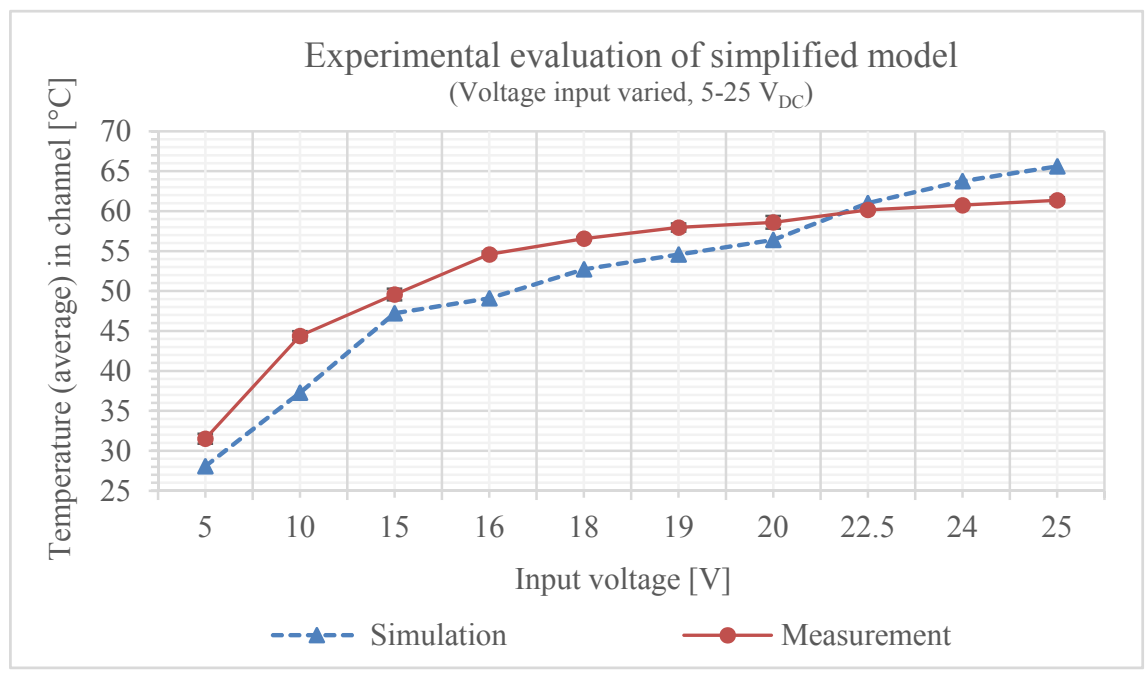

Figure 2: $\quad$ Evaluation of model -1 . 
Measurements were taken for 45 minutes, and the same time period was simulated. Solution time of this model was 17 minutes 24 seconds ( \pm 8 seconds) on a PC with a Core i5-4570 CPU and 16 GB RAM for a single set of input parameters.

In the experimental setup, the temperature probe was positioned in the middle of the channel, whereas in the simulation, temperature was averaged for the volume of the channel and over the time period of the measurements. The comparison of simulated and measured data points is shown in Figure 2. The average estimation error of the model was $3.59 \pm 1.66^{\circ} \mathrm{C}$ degrees over the given range of input voltages.

\subsection{Evaluation of the extended model}

For this evaluation, the LAMP (loop-mediated isothermal amplification) reaction, an isothermal nucleic acid amplification method was taken into account. This is a highly sensitive and specific amplification method particularly suitable for use in molecular diagnostics, developed by Eiken Chemical Co. (Nagamine et al. [8]), which requires a $60-65^{\circ} \mathrm{C}$ temperature range to be maintained for $15-60$ minutes for a successful amplification of DNA samples. The extended model was designed to provide a better approximation of reality, given that the internal structure of the heating element is known in detail. This extension takes into account the current distribution, and therefore yields a more precise definition of heat sources inside the heating element. Having the aforementioned isothermal application in mind, the target temperature range $60-65^{\circ} \mathrm{C}$ was taken into account for the evaluation, which was possible to reach experimentally at $24 \mathrm{~V}_{\mathrm{DC}}$ input, so this voltage was used for comparison.

The extended model was solved using the Joule Heating interface of COMSOL $^{\circledR}$ Multiphysics Version 4.4 using the same geometry and material composition as for the simplified model. The experimental setup generated the required heat output at $24 \mathrm{~V}$ input, therefore this voltage was used as a basis for comparison between modelling and measurement results. Solution time of this model was 11 minutes 50 seconds ( \pm 16 seconds) on a PC with a Core i5-4570 CPU and 16 GB RAM for a single set of input parameters.

Temperature output was measured in a similar manner as during the evaluation of the simplified model. The extended model estimates temperature output around the set point (reached after $1800 \mathrm{~s}$ in both cases) with an error of $0.31 \pm 0.17^{\circ} \mathrm{C}$. Comparison of results is shown in Figure 3.

The heat generation of the experimental setup was more rapid than that of the model, which were probably attributable to the differences in model geometry. The heating elements could not be disassembled without causing irreversible damage, and the patent of Bohlender and Buchlaub [9] describing the internal structure did not include dimensions, so the geometry of the simulated model was approximate. The extended model is better used with heating elements, the internal structure of which is known in detail. 


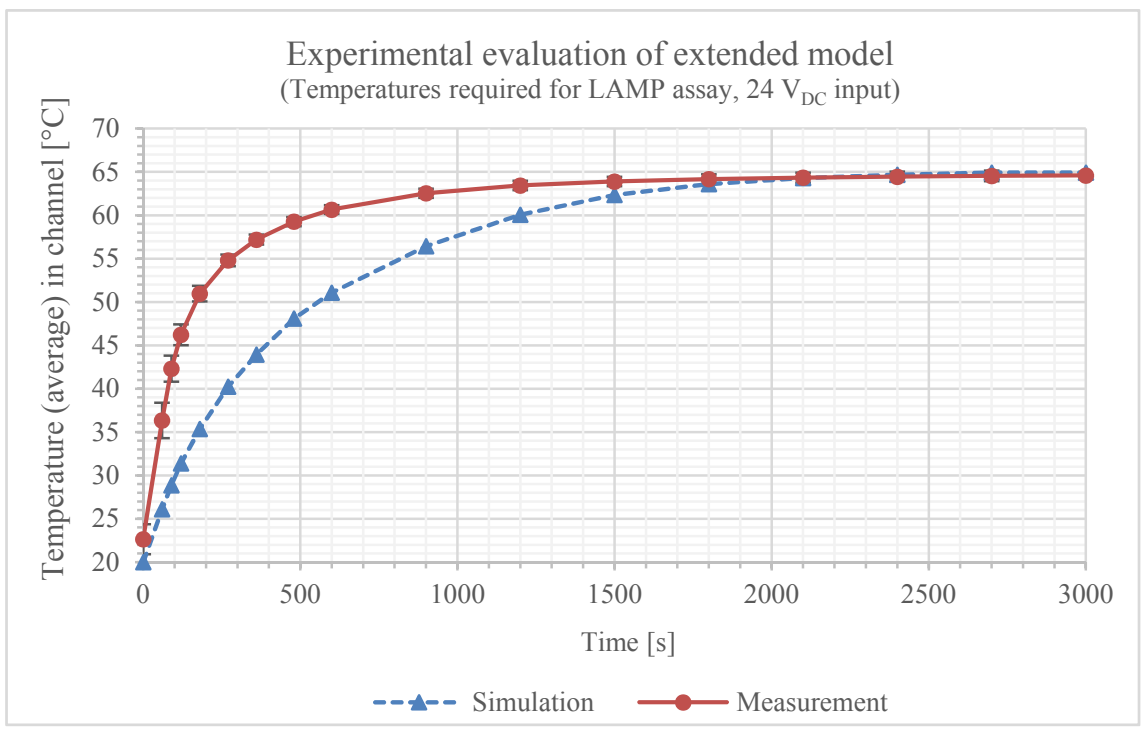

Figure 3: $\quad$ Evaluation of model -2 .

\section{Conclusions}

A finite element model was proposed for the modelling of self-regulating resistive heating elements for applications in microheating solutions for disposable molecular diagnostics that relies only on the temperature coefficient of resistance (resistivity) for characterising the heating element. A simplified and an extended version of the model were presented and evaluated for an experimental setup that imitated a disposable diagnostics platform with a replaceable microfluidic cartridge (chip) and a commercially available self-regulating heating element. The simplified model approximated the temperature output of the experimental setup with an error of $3.59 \pm 1.66^{\circ} \mathrm{C}$ degrees over a range of various input voltages and a time range of 45 minutes, whereas the extended model approximated temperature output at $24 \mathrm{~V}$ input with an error of $0.31 \pm 0.17^{\circ} \mathrm{C}$ after the set point was reached.

The results discussed in this paper are preliminary and show an early stage of development. Nevertheless, proof was given that the proposed models are capable of estimating the heat output of microheating solutions in diagnostic devices, and as the in-house development of heating solutions proceeds, the precision of these estimations are bound to improve significantly.

\section{Acknowledgements}

The authors wish to thank for the support of the Sächsische AufbauBank provided under project SAB\#100144699, and the Estonian Research Council provided under research projects SF0140061s12, ETF8592 and IUT19-11, and the 
Foundation Archimedes through the Centre of Excellence CEBE (TK05U01) for supporting technology-oriented scientific projects in Estonia. Additionally, the authors would like to express their gratitude to Jüri Oleitšuk for the manufacturing of test structures.

\section{References}

[1] Wyzkiewicz, I., Grabowska, I., Chudy, M., Brzozka, Z., Jakubowska, M., Wisniewski, T. \& Dybko, A., Self-regulating heater for microfluidic reactors, Sens. Actuators B Chem., vol. 114, no. 2, pp. 893-896, Apr. 2006.

[2] Shioi, R., Umeya, K., Yonezuka, K. \& Senzaki, H., Heating element made of PTC ceramic material, Tdk Electronics Company Limited, 1981.

[3] Huybrechts, B., Ishizaki, K., \& Takata, M., The positive temperature coefficient of resistivity in barium titanate, J. Mater. Sci., vol. 30, no. 10, pp. 2463-2474, 1995.

[4] Meier, A. von., Electric power systems a conceptual introduction, Hoboken, N.J.: IEEE Press, Wiley-Interscience, 2006.

[5] Rood, P., A visual method of showing the high temperature coefficient of resistance of metals as compared with alloys, J. Opt. Soc. Am., vol. 16, no. 5, p. 357, Sep. 1928.

[6] Kandlikar, S. G., Heat transfer and fluid flow in minichannels and microchannels, Elsevier, 2006.

[7] Griffiths, D. J., Introduction to electrodynamics, Prentice Hall, 1999.

[8] Nagamine, K., Hase, T. \& Notomi, T., Accelerated reaction by loop-mediated isothermal amplification using loop primers, Mol. Cell. Probes, vol. 16, no. 3 , pp. 223-229, Jun. 2002.

[9] Bohlender, F. \& Buchlaub, N., PTC heating resistor, David \& Baader DBK Spezialfabrik elektrischer Apparate und Heizwiderstände GmbH, 1989. 